\title{
EMERGENCE OF GRANULAR-SIZED MAGNETIC BUBBLES THROUGH THE SOLAR ATMOSPHERE. II. NON-LTE CHROMOSPHERIC DIAGNOSTICS AND INVERSIONS
}

\author{
Jaime de la Cruz Rodríguez ${ }^{1}$, Viggo Hansteen ${ }^{2}$, Luis Bellot-Rubio ${ }^{3}$, and Ada Ortiz ${ }^{2}$ \\ ${ }^{1}$ Institute for Solar Physics, Department of Astronomy, Stockholm University, Albanova University Center, SE-10691 Stockholm, Sweden; jaime@ astro.su.se \\ ${ }^{2}$ Institute of Theoretical Astrophysics, University of Oslo, P.O. Box 1029 Blindern, NO-0315 Oslo, Norway \\ ${ }^{3}$ Instituto de Astrofísica de Andalucía (CSIC), Apartado de Correos 3004, E-18080 Granada, Spain \\ Received 2015 March 12; accepted 2015 August 3; published 2015 September 8
}

\begin{abstract}
Magnetic flux emergence into the outer layers of the Sun is a fundamental mechanism for releasing energy into the chromosphere and the corona. In this paper, we study the emergence of granular-sized flux concentrations and the structuring of the corresponding physical parameters and atmospheric diagnostics in the upper photosphere and in the chromosphere. We make use of a realistic 3D MHD simulation of the outer layers of the Sun to study the formation of the Ca II 8542 line. We also derive semi-empirical 3D models from non-LTE inversions of our observations. These models contain information on the line-of-sight stratifications of temperature, velocity, and the magnetic field. Our analysis explains the peculiar Ca II 8542 A profiles observed in the flux emerging region. Additionally, we derive detailed temperature and velocity maps describing the ascent of a magnetic bubble from the photosphere to the chromosphere. The inversions suggest that, in active regions, granular-sized bubbles emerge up to the lower chromosphere where the existing large-scale field hinders their ascent. We report hints of heating when the field reaches the chromosphere.
\end{abstract}

Key words: line: formation - polarization - radiative transfer - Sun: chromosphere - Sun: magnetic fields

\section{INTRODUCTION}

Currently, one of the foremost questions in solar physics concerns the mechanisms that transport and release nonthermal energy into the outermost layers of the Sun (e.g., Mariska 1992; Klimchuk 2006). The answer to these questions is evidently connected with understanding the fine structuring observed in the chromosphere, because the conversion of nonthermal energy into thermal energy occurs at very small spatial scales (e.g., De Pontieu et al. 2014a; Hansteen et al. 2014; Tian et al. 2014). Magnetic fields and magnetic reconnection play important roles in heating the chromosphere and corona (e.g., Galsgaard \& Nordlund 1996; Gudiksen \& Nordlund 2005). Reconnection can occur as a result of the braiding of the previously existing magnetic field by photospheric motions, but can be strongly enhanced when a new magnetic flux emerges through the photosphere (Galsgaard et al. 2007). The emergence of magnetic flux into the outer layers of the Sun also plays an important role in the formation of sunspots and active regions, and is an integral part of the solar cycle (Schmieder et al. 2014).

In recent years, a number of observational studies have aimed to describe how small-scale magnetic fields emerge in the outer layers of the Sun and to establish observational constraints on the shape, field strength, and speed of ascent through the photosphere and lower chromosphere (Martínez González \& Bellot Rubio 2009; Schmieder et al. 2014). In the photosphere, great attention has been given to magnetic flux emergence in the quiet Sun because the ubiquity of the latter would translate into large amounts of magnetic flux being deposited into the outer layers of the atmosphere (see recent studies by Lites et al. 2008; Beck \& Rezaei 2009; Danilovic et al. 2010; Gošić et al. 2014, and references therein).

In regions with relatively high magnetic activity, and therefore stronger polarization signatures, observers have focussed on the coupling between the photosphere and the lower chromosphere and have studied the connectivity of the emerging flux (Guglielmino et al. 2010; Ortiz et al. 2014). However, not much work has been done using chromospheric diagnostics, probably because non-LTE/non-equilibrium conditions make it very difficult to translate the observed intensities into the underlying physical state of the atmosphere (see, e.g., Socas-Navarro et al. 2000; Leenaarts et al. 2012).

Realistic radiative numerical MHD simulations of flux emergence have, in most cases, been restricted below the upper photosphere (e.g., Cheung et al. 2008; Tortosa-Andreu \& Moreno-Insertis 2009) because non-LTE radiative losses must be included to reproduce chromospheric conditions. Using the non-LTE method developed by Skartlien (2000), MartínezSykora et al. (2008, 2009), studied for the first time the emergence of magnetic flux in a simulation including a photosphere, chromosphere and corona. They investigated the chromospheric and coronal response to the emergence of magnetic flux, reporting the formation of cold magnetic bubbles in the upper photosphere that expel chromospheric oscillations and push the transition region and the corona to heights much greater than $2 \mathrm{Mm}$.

A similar scenario is described by Ortiz et al. (2014; hereafter Paper I), who compared Ca II IR observations with the atmospheric parameters of a numerical simulation. They find observational evidence of these cold bubbles, which had been previously found in simulations by Martínez-Sykora et al. (2008, 2009). The ascent of a magnetic bubble from the photosphere into the chromosphere was described using very high spatial-resolution data in the $\mathrm{Fe}_{\mathrm{I}} \lambda 6301 / \lambda 6302$ and the Ca II $\lambda 8542$ lines. Furthermore, in Paper I the authors also presented photospheric LTE inversions from the $\mathrm{Fe}_{\mathrm{I}}$ data set and a qualitative study of the $\mathrm{Ca}$ II data, including a weak-field approximation analysis to infer the strength of the magnetic field in the chromosphere.

In this paper, we investigate the emergence of small-scale magnetic flux from the photosphere into the chromosphere, continuing the study initiated by Ortiz et al. (2014). Our 
analysis is prompted by our desire to know the structuring of physical parameters in the upper photosphere and lower/ middle chromosphere during the emergence of a cold magnetic bubble. We also wish to understand the peculiar shape of the Ca II $\lambda 8542$ intensity profiles observed in the flux emergence events, with a bump in the red wing that looks like an emission peak. The former are obtained by performing non-LTE inversions of high spatial-resolution observations, whereas the latter is investigated using a realistic 3D MHD simulation of the outer layers of the Sun.

In Section 2 we describe the processing of the observations, the inversions, and the computation of synthetic profiles from the 3D MHD simulations. In Section 3 we analyze the inverted models and we discuss the formation of the Ca II 8542 line during the flux emergence, using the 3D simulation. Our conclusions are summarized in Section 4.

\section{DATA}

\subsection{Observations with the SST}

We analyze the same data set that was used in Paper I. Our observations of AR11024 were acquired on 2009 July 05 starting at 09:48 UT at coordinates $\mathrm{S} 27^{\circ}, \mathrm{W} 12^{\circ}$. The data were taken with the Swedish $1 \mathrm{~m}$ Solar Telescope (SST, Scharmer et al. 2013) and the CRisp Imaging Spectro-Polarimeter (CRISP, Scharmer et al. 2008) in the Ca II $\lambda 8542 \AA$ line (hereafter $\lambda 8542$ ). We sequentially acquired narrow band images sampling the $\lambda 8542$ profile at 17 line positions in steps of $100 \mathrm{~m} \AA$ between $\Delta \lambda= \pm 800 \mathrm{~m} \AA$ from line center. An additional point was measured at $\Delta \lambda=2400 \mathrm{~m} \AA$.

The data were reduced using the CRISPRED pipeline (de la Cruz Rodríguez et al. 2015). The images were restored with multi-frame-multi-object-blind-deconvolution techniques (MOMFBD, van Noort et al. 2005), and residual seeing distortions within the line scan have been compensated using methods described in Henriques (2012).

It is not possible to reach the continuum of the $\lambda 8542$ line with CRISP because the prefilter is not wide enough. Therefore, to scale our observations relative to the continuum of the quiet-Sun spatial average at disk-center, we use a 3D HD simulation (de la Cruz Rodríguez et al. 2011). The idea is to compute a simulated spatio-temporal average of quiet-Sun spectra at $\mu=0.87$ (where our observations were acquired). Then we normalize the average intensity profile by the continuum intensity at $\mu=1$. Finally, we scaled our observations in such a way that the average quiet-Sun spectrum at $\Delta \lambda=2.4 \AA$ has the same intensity as the normalized synthetic average profile. The same technique was used in Paper I and by Watanabe et al. (2012).

The absolute wavelength calibration is performed using a spatio-temporal average from a patch of quiet Sun, such that the line center is at rest. It is hard to find velocity references in the solar atmosphere, particularly in the chromosphere. The wavelength coverage of our observations do not allow to compute a photospheric convective blushift that could be used as a reference for Doppler velocities (de la Cruz Rodríguez et al. 2011).

In Paper I we described the peculiar intensity profile of the $\lambda 8542$ line during the flux emergence event. Figure 1 illustrates a time series of the profile within the region of interest, indicated in the corresponding images with a white square. The

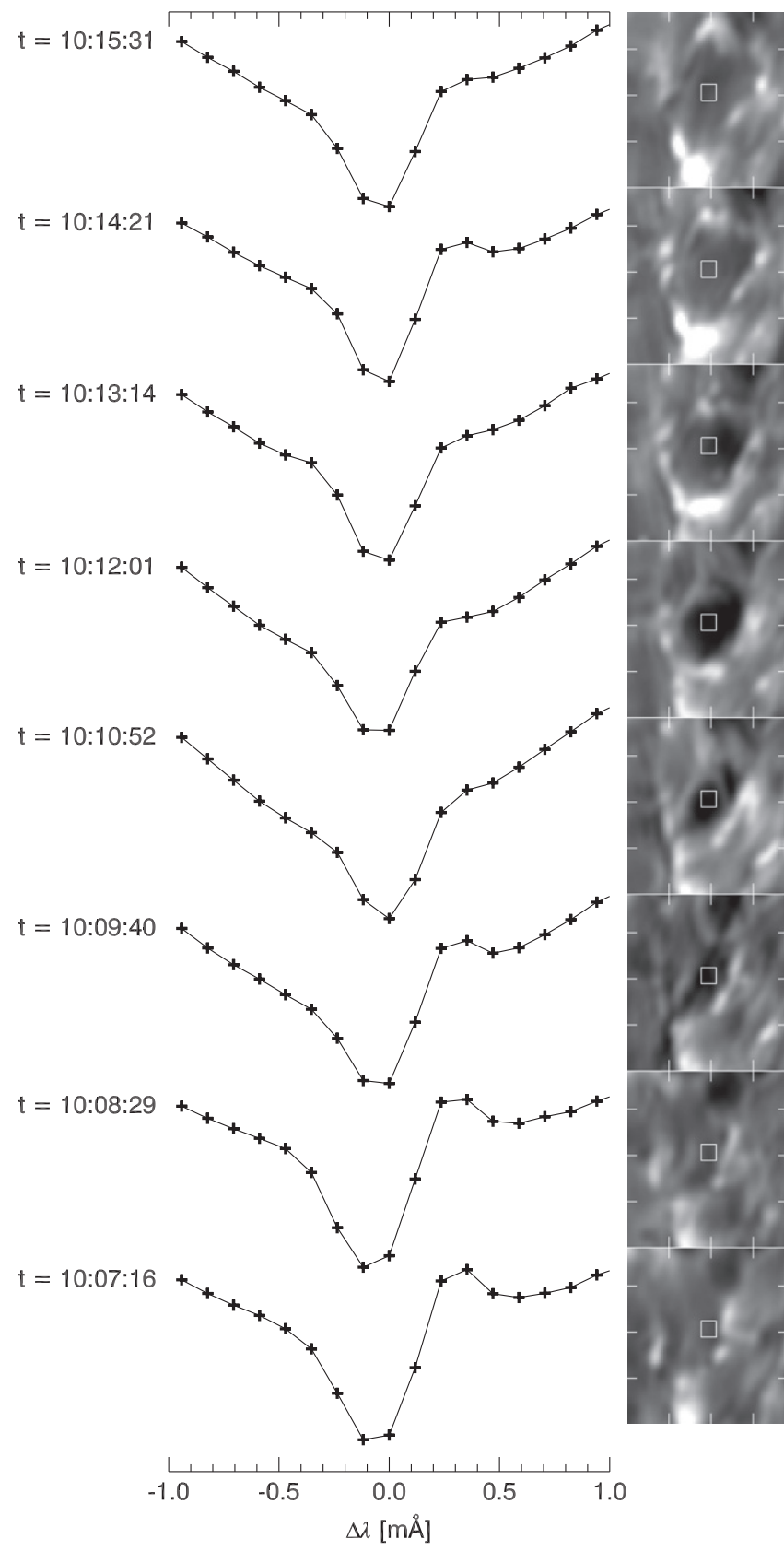

Figure 1. From bottom to top, time evolution of the $\lambda 8542$ spectra, spatially averaged within the small white box $(7 \times 7$ pixels $)$ indicated in the filtergrams on the right. The tick-mark separation is $1^{\prime \prime}$ in the images.

profile has three distinctive features compared with a spatial quiet-Sun average.

1. The line center is clearly blueshifted.

2. Close to the line center, the line profile is strongly asymmetric, with a very sharp gradient on the red side and a much more extended blue side.

3. At approximately $+250 \mathrm{~m} \AA$ there is an emission feature that is not similarly visible on the blue wing.

The degree of asymmetry and the exact strength of the three features enumerated above suffer slight changes over time, but the overall shape of the profile is persistently similar. In our analysis, we will try to understand the formation of the $\lambda 8542$ line and the atmospheric parameters that are responsible for these features. 


\subsection{Non-LTE Calculations}

We use the non-LTE inversion code NicOLE to compute inversions in the $\lambda 8542$ line (Socas-Navarro et al. 2015). The code iteratively modifies the physical parameters of a guessed model atmosphere to reproduce observed spectra (SocasNavarro et al. 2000). NiCOLE solves the non-LTE problem as described in Socas-Navarro \& Trujillo Bueno (1997) assuming statistical equilibrium, which is reasonable for $\mathrm{Ca}$ II lines (Wedemeyer-Böhm \& Carlsson 2011). Our atom model consists of five bound levels plus an ionization continuum, similar to that used by Leenaarts et al. (2009). The atomic data used in this study has been extracted from the VALD database (Piskunov et al. 1995).

NiCOLE assumes Zeeman-induced polarization to compute the full-Stokes vector, neglecting scattering polarization and the Hanle effect. This assumption works fine in regions with relatively strong magnetic fields, but in the quiet Sun all these effects must be considered to model Stokes $Q$ and $U$ observations (Manso Sainz \& Trujillo Bueno 2010; Carlin et al. 2012; Carlin \& Asensio Ramos 2015).

The radiative transfer equation is solved using a piece-wise cubic Bezier spline interpolant both to calculate the non-LTE populations (assuming unpolarized light) and to compute the final polarized formal solution (Auer 2003; de la Cruz Rodríguez \& Piskunov 2013).

The spatially averaged quiet-Sun spectrum of the $\lambda 8542$ line shows a reversed C-shape bisector (Uitenbroek 2006b; Chae et al. 2013; Pietarila \& Harvey 2013). Only recently, Leenaarts et al. (2014) realized that the asymmetry in solar observations is produced by the presence of different $\mathrm{Ca}$ II isotopes, apparently a well-known phenomenon in chemically peculiar stars (Castelli \& Hubrig 2004; Cowley \& Hubrig 2005; Cowley et al. 2007; Ryabchikova et al. 2008).

We have included the effect of isotopic splitting in our inversions using a simple approximation. First, the non-LTE problem is solved with a regular ${ }^{40} \mathrm{Ca}$ atom. Once the atom population densities are known, we compute a final formal solution of the radiative transfer equation, assuming that the absorption coefficient is a weighted sum of Voigt profiles, each of them centered at the rest-wavelength of the line for each isotope. In our case, the weight is proportional to the relative abundance of each isotope (Carlsson 1986).

Our observations are under-sampled by almost a factor $\times 2$ in the spectral dimension, a necessary trade-off to keep the cadence sufficiently high while observing three spectral lines. This situation is less than ideal for inversions, where critically sampled observations constrain the solution much better. Therefore we have run a set of tests to assess the optimal combination of nodes that allows us to reproduce the observed profiles with the lowest number of degrees of freedom that is possible. The inversions are performed in several cycles to improve the convergence (Ruiz Cobo \& del Toro Iniesta 1992): during the first cycle, a reduced number of nodes is considered. Once the solution cannot be improved further, a second cycle is started from that point with more nodes. Table 1 summarizes the number of nodes used in each of the cycles.

In our first tests with the number of nodes, we realized that placing the nodes equidistantly along the depth-scale is not an optimal choice. After some trial and error, we found that we obtain much better fits, at least whenever there is a node placed at $\log \tau_{500} \sim-4.5$ both in temperature and in velocity.
Table 1

Number of Nodes Used in Each Cycle of Our Inversions for Temperature, Line-of-sight (LOS) Velocity, and Longitudinal Magnetic Field $\left(B_{\text {Long }}\right)$

\begin{tabular}{lccc}
\hline \hline & Cycle 1 & Cycle 2 & Cycle 3 \\
\cline { 2 - 4 } Temp & 4 & 5 & 5 \\
$v_{\text {LOS }}$ & 2 & 4 & 4 \\
$B_{\text {long }}$ & 0 & 0 & 1 \\
\hline
\end{tabular}

We have modified NiCOLE to compute the response functions (see definitions in Section 3.2) using centered numerical derivatives instead of the default derivatives based on oneside perturbations of the model parameters. This makes the code roughly 33\%-50\% slower, but it greatly improves the convergence of the inversion that now reaches more consistently similar values of $\chi^{2}$ from different randomizations of the initial model parameters. Additionally, inspired by van Noort (2012), we have removed some inversion noise ${ }^{4}$ by inverting the data once, horizontally smoothing the parameters of the model at the locations of the nodes, and then restarting the inversion again. This trick only seems to help in pixels where the inversion clearly got stuck in a local minimum.

\subsection{Synthetic Observation from the 3D Simulation}

To study the formation of the Ca II $\lambda 8542$ line we use a 3D magnetohydrodynamical simulation performed with the BIFROST code (Gudiksen et al. 2011), which has also been used in Paper I. Synthetic full-Stokes $\lambda 8542$ observations have been calculated with NICOLE, as described in Section 2.2.

The simulation includes the upper convection-zone, photosphere, chromosphere, and corona covering a physical domain that extends vertically from $z_{0}=-2.6 \mathrm{Mm}$ to $z_{1}=14 \mathrm{Mm}$ from the surface of the photosphere. Horizontally, the simulation covers approximately $24 \times 24 \mathrm{Mm}$. This domain is discretized in a grid of $504 \times 504 \times 496$ points, with a horizontal step of $47.6 \mathrm{~km}$ and a vertical step of $20 \mathrm{~km}$ in the radiative zone $(-400 \leqslant z \leqslant 5000 \mathrm{~km})$. In the convection zone and corona the vertical distance between grid points becomes larger with increasing scale height.

The calculations are performed with periodic boundary conditions in the horizontal plane, and open boundary conditions at the top and at the bottom. At the beginning of the simulation, the magnetic flux is injected at the bottom boundary. The injected field is a flux sheet with no twist oriented along the $y$-axis from $x_{0}=4 \mathrm{Mm}$ to $x_{1}=16 \mathrm{Mm}$ with strength $B=3300 \mathrm{G}$. This injection continues for $1 \mathrm{hr}$ 45 minutes before being turned off. The field rises steadily through the convection zone to the photosphere before stalling there after about an hour. Eventually the field breaks through the photosphere, in certain locations, and into the chromosphere, forming expanding bubbles of magnetic field as described in greater detail in Paper I. Later stages of this simulation were used to study small flares by Archontis \& Hansteen (2014).

We note that compared to the latest runs performed with BIFROST, this simulation does not include the effect of collisions between neutrals and ions that can influence the temperature stratification in the chromosphere (Khomenko \& Collados 2012; Martínez-Sykora et al. 2012; Martinez-Sykora

\footnotetext{
4 Noise in the inferred 2D maps partly from non-smooth convergence of adjacent pixels.
} 


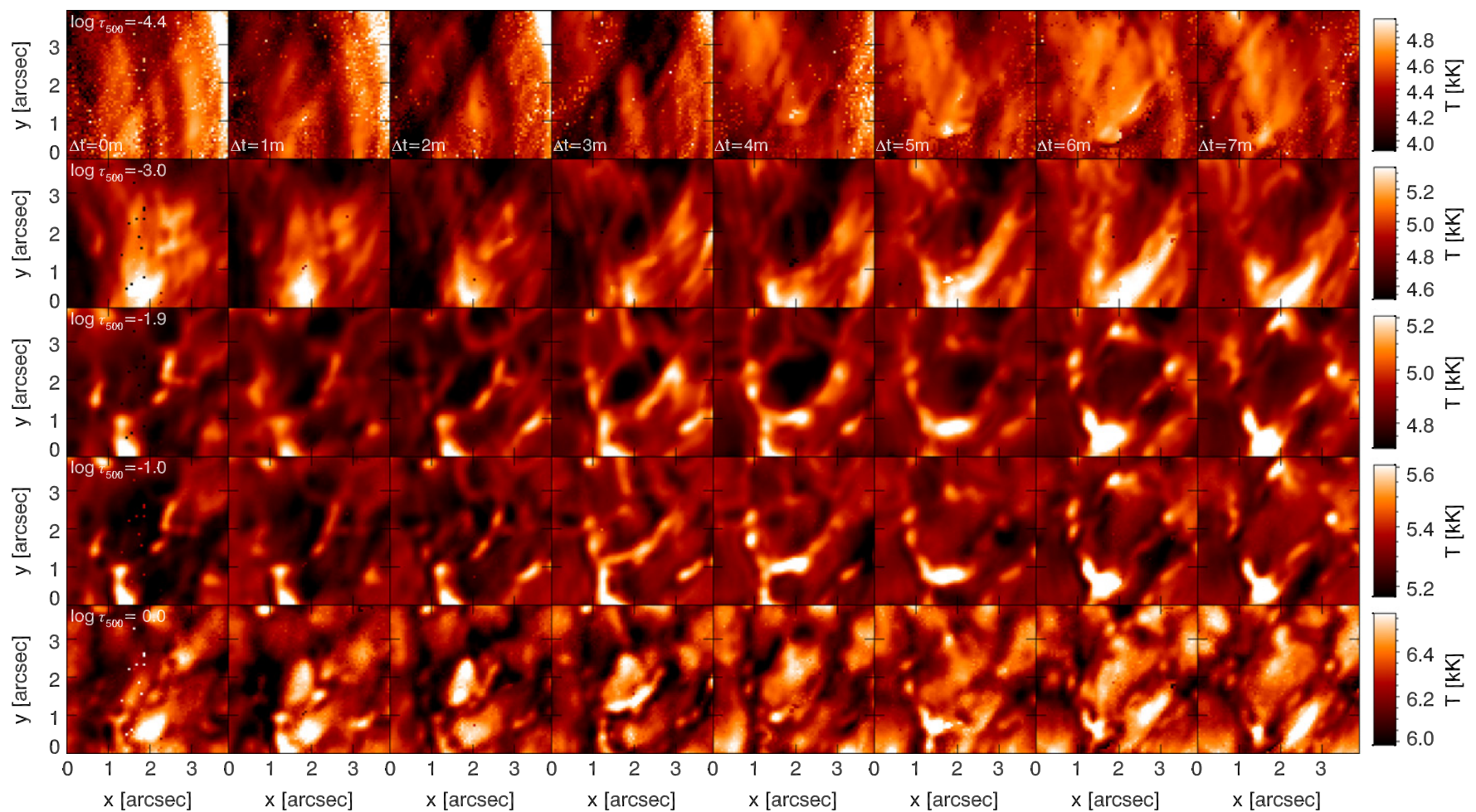

Figure 2. Temporal evolution of the temperature inferred with a non-LTE inversion. From left to right, the panels show consecutive time steps from our time series. From bottom to top, the panels illustrate the inferred temperature at iso-log $\tau_{500}$ surfaces in the model. $\Delta t=0$ corresponds to 10:07:16 UT.

et al. 2015) or the effect of hydrogen non-equilibrium ionization that can increase the amount of free electrons in the chromosphere, affecting the opacity of chromospheric lines (Leenaarts et al. 2007).

\section{ANALYSIS}

\subsection{D Models from Non-LTE Inversions}

In this section we discuss the results of the non-LTE inversions of the observed Ca II $\lambda 8542$ Stokes profiles. We will focus on the inferred temperature and line-of-sight velocity maps.

Figure 2 illustrates the inferred temperature as a function of time at $\log \tau_{500}=-4.4,-3.0,-1.9,-1.0,0.0$. We note that the panels at $\log \tau_{500}=0.0$ may be an extrapolation of the model in the upper layers because our observations do not reach the continuum. Even so, those deep photospheric layers show typical granulation patterns with hot granules, colder intergranules, and hot bright-points (though geometrically the latter are probably located in deeper layers than the former, see Steiner et al. 2001).

At $\log \tau_{500}=-1.0$ the granulation pattern is reversed showing hotter intergranular lanes than granules (Cheung et al. 2007). Here the footpoints of the bubble are clearly visible, but otherwise, there are no signatures of the bubble in the temperature map. Only at $\log \tau_{500} \sim-1.9$, in the center of the bubble above the granule, does the temperature show a significant drop compared to the surroundings, especially between $\Delta t=1$ minute and $\Delta t=4$ minute. Later on, the temperature rises again in the area where the bubble appeared.

At $\log \tau_{500}=-3.0$ there is a dramatic change in the structuring of the temperature, and features that resemble fibrils start to be visible. Therefore we associate this panel with the lower chromosphere. The magnetic bubble reaches these layers with a temporal delay of roughly 2 minutes compared to $\log \tau_{500}=-1.9$, similarly to what was reported in Paper I from the analysis of images at different line positions.

Above the lower footpoint (in the image) there are signatures of heating, quite localized until $\Delta t=4$ minute when they extend around the bubble. We speculate that this heating can be produced by three different processes:

1. by magnetic interaction of the footpoints with the existing magnetic canopy of fibrils.

2. by wave dissipation, assuming that waves are channeled through the footpoints and persistently release energy into the chromosphere.

3. by Joule heating at the boundary of the magnetic bubble, where steep gradients in the magnetic field drive electrical currents (Solanki et al. 2003).

Unfortunately, we would need very accurate estimates of the magnetic field at these locations to study whether the brightenings are due to reconnection and/or to estimate the location and effect of electrical currents in our maps. Therefore, this aspect must be clarified in future studies, hopefully with higher sensitivity data.

At $\log \tau_{500}=-4.4$ our inversions show a more homogeneous temperature structuring, with no signature of the emerging field. This event is right at the end of the time series, so we cannot confirm whether the bubble manages to reach the middle/upper chromosphere: in our data set it does not. The canopy may hinder the ascent of the bubble and we find evidences of magnetic interaction between the emerging flux and the existing field especially at the boundary of the bubble. Interestingly, the entire region in the temperature panels at 


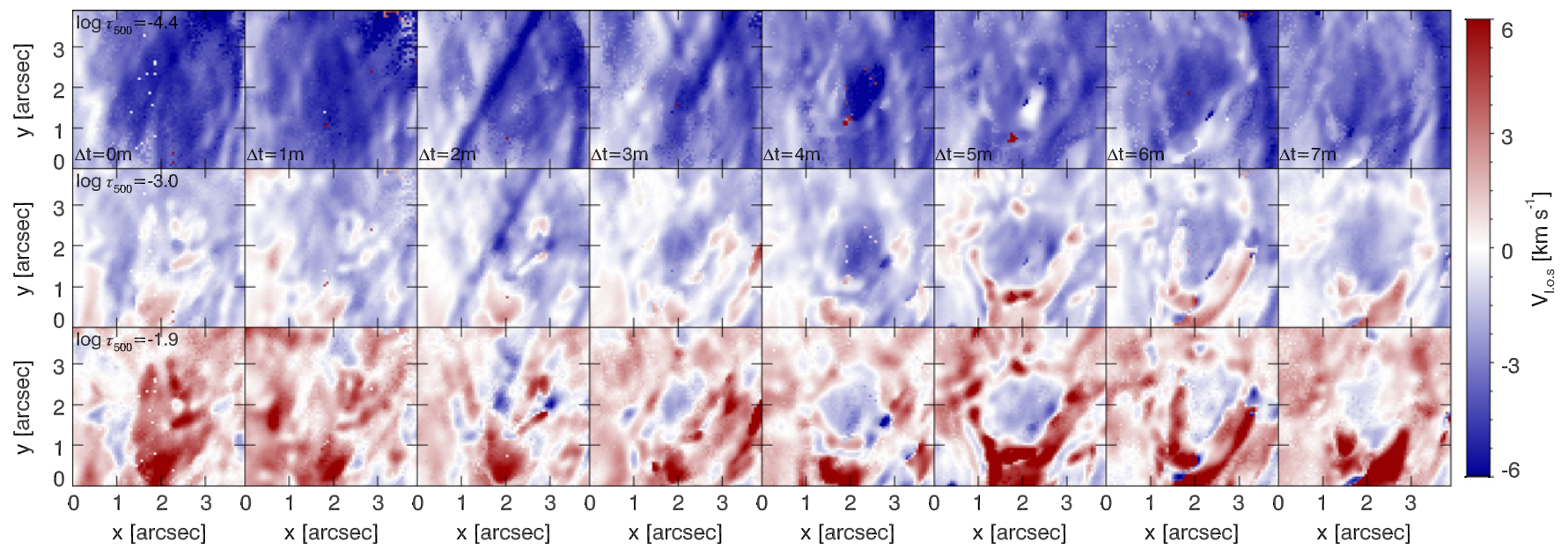

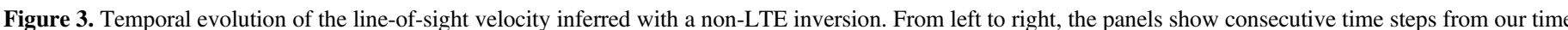
series. From bottom to top, the panels illustrate the inferred temperature at iso-log $\tau_{500}$ surfaces in the model. $\Delta t=0$ corresponds to $10: 07: 16$ UT.

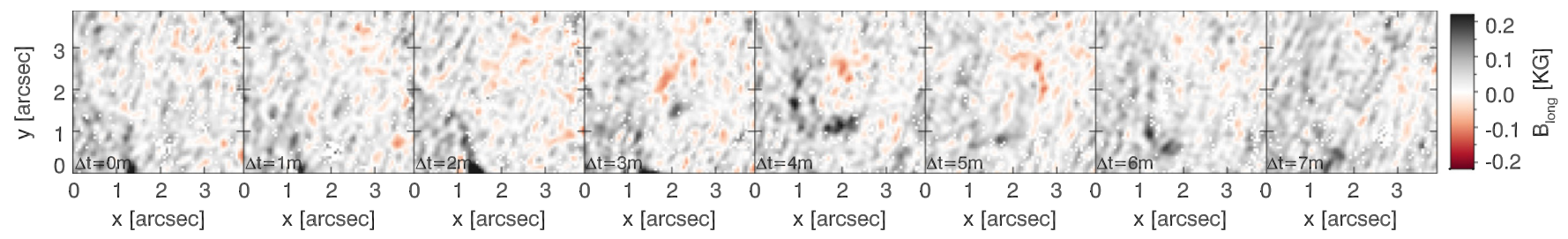

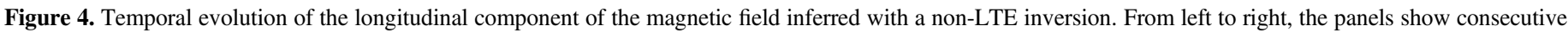
time steps from our time series. $\Delta t=0$ corresponds to 10:07:16 UT.

$\log \tau_{500}=-4.4$ seems to get slightly warmer by the end of the time series.

It is also clear that the apex of the bubble must be located between $\log \tau_{500}=-3.0$ and $\log \tau_{500}=-4.4$ in our observations. We note that the panels in Figure 2 may show reduced temperature contrast due to 3D non-LTE effect (see de la Cruz Rodríguez et al. 2012) or to stray light in the observations.

Similarly, 2D maps of inferred velocities are presented in Figure 3. Our results in the deepest layers of the inverted model seem to be an extrapolation of the velocity field around $\log \tau_{500} \approx-1.5$. We are not surprised because the Lorentzian (photospheric) wings are likely to be much more sensitive to changes in temperature than to changes in the velocity field, since the intensity gradient is not very steep. Close to the line center, the profiles carry more complete information of the velocity field.

Our inversion results at $\log \tau_{500}=-1.9$ are very similar to those inferred using Fe I lines in Paper I. Gas flows up in the center of the bubble and drains down at the sides. These downflows coincide with the location of intergranular lanes of photospheric granulation. At $\log \tau_{500}=-3.0$, the imprint of these downflows in the surroundings has almost vanished, but in some locations they are visible at the end of the series, when the bubble has reached high enough in the atmosphere. Finally, at $\log \tau_{500}=-4.4$ the images are dominated by the canopy of fibrils, which appear up-flowing along our line of sight. The entire region seems to be moving upward with speeds of -6 $\mathrm{km} \mathrm{s}^{-1}$.

We now discuss the inferred longitudinal component of the magnetic field, shown in Figure 4. The maps at $\Delta t=0,1,2$ minutes do not show any features at the location of the bubble. However, from $\Delta t=3$ minutes to $\Delta t=5$ minutes two patches of opposite polarities appear and slowly separate during the ascent of the bubble. From $\Delta t=6$ minutes, one of the footpoints is still visible at the bottom of the image. The inferred longitudinal field has amplitudes peaking $\pm 140 \mathrm{G}$, which seems to lay just above our sensitivity threshold. This inversion has been performed with a constant magnetic field since the polarization signals are too small to attempt to infer gradients.

Initially we were surprised to see these opposite polarity patches. In Paper I, we used the far wings and the inner core of the 8542 line to estimate the photospheric and chromospheric fields, respectively. The results show half-moon shaped footpoints in the photosphere (with opposite polarities), whereas we only detected signals in a very small patch above the footpoints in the chromosphere, but unfortunately not in the interior of the bubble. We suspect this is due to the too restrictive wavelength ranges we used to compute the weakfield approximation both at the core and at the wings, skipping those parts of the line profile where the bulk of the signals are contained.

Now we discuss the quality of the fits to the observed profiles that are reasonably good, but not perfect. Figure 5 shows an example from the upper opposite polarity patch, at $\Delta t=3$ minutes. The general aspect of the Stokes $I$ profile is reproduced by the inversion code, but the sharpest features in the observations appear smoothed in the inversion, an expected result from the discretization of the nodes in the inversion (de la Cruz Rodríguez et al. 2012): the location and separation of the nodes are not ideal to capture sharp gradients in the atmosphere. The Stokes $V$ profiles are very noisy, but the code seems to find a solution that is compatible with the observations assuming a constant magnetic field.

We focus now on the strongly tilted blue wing of the profile (see Figure 1). As we described in Paper I, the imprint of the 


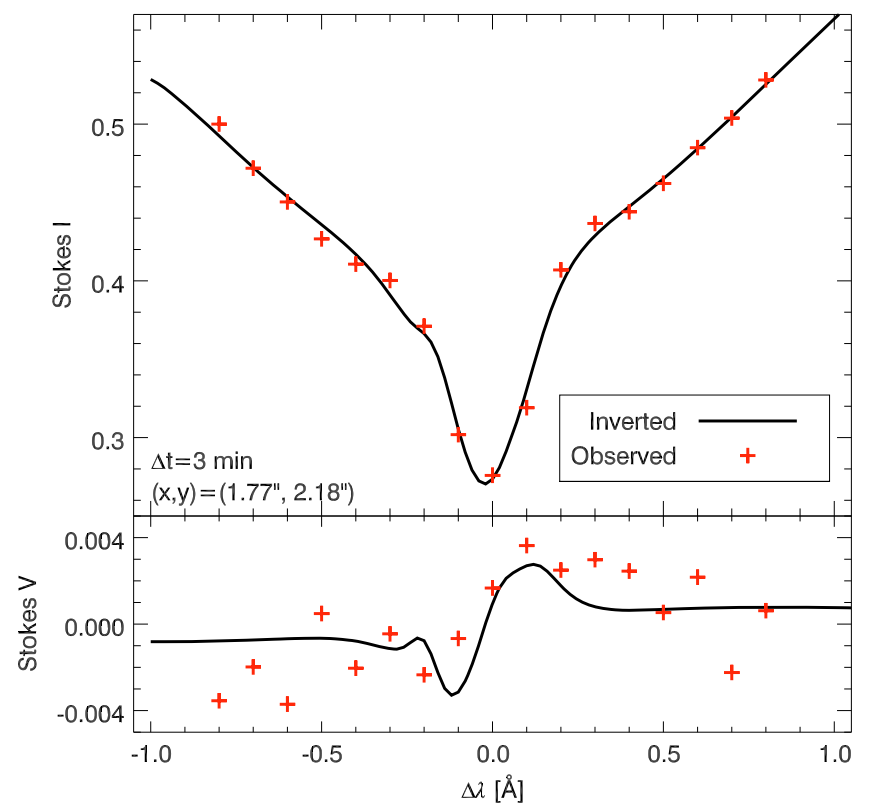

Figure 5. Exemplary observed (red crosses) and best-fit (black solid) profiles from the center of the magnetic bubble at $\Delta t=3$ minute, at the upper opposite polarity patch. Stokes $I$ (top) and Stokes $V$ (bottom). The profiles have been normalized by the continuum intensity of a quiet-Sun average at that wavelength $\left(\left\langle I_{\mathrm{c}}\right\rangle\right)$.

bubble is more prominent in the blue wing of the line. This effect is particularly visible from $\Delta \lambda=-800 \mathrm{~m} \AA$ to $\Delta \lambda=-300 \mathrm{m \AA}$. As we move to the center of the line, the monochromatic images are dominated by fibrils. Therefore, the profile is sampling to different regimes in the atmosphere, the bubble in the outer photospheric wings, which causes a strong asymmetry, and a canopy of fibrils close to line center. The exact steepness of the velocity gradient and the projection of the canopy of fibrils along the line of sight will define how strong these effects are and how the line center is shifted relative to the wings.

\subsection{The Formation of the $\lambda 8542$ Line within an Emerging Bubble}

The advantage of the MHD simulation is that we have all of the thermodynamical properties in hand, making it possible to study the formation of the line. We have been puzzled by the peculiar shape of the line profiles inside the bubble. To find an explanation for such profiles, our first attempt was to inspect the properties of the 3D simulation.

Figure 6 shows synthetic images in the Ca II $\lambda 8542$ line at different wavelengths, for one time-step of the simulation. In the left-most panel we illustrate the line-core intensity for each pixel, and in the consecutive panels, monochromatic images at $\Delta \lambda=-100,-75,0,75 \mathrm{~m} \AA$ relative to rest wavelength. In the first panel, we have effectively removed intensity fluctuations due to Doppler motions, and therefore, the dark circular shape of the flux emerging region becomes clearer. In the monochromatic panels, the intensity also changes due to Doppler motions, but it is clear that spectra within the emerging region are blueshifted, and therefore, that it is darker at blue wavelengths. This is similar to what is observed, as described in Paper I. However, the synthetic profiles are significantly narrower than those from real observations, with a much steeper Gaussian core than in the observations.
Therefore, the imprint of Doppler shifts is quite strong in the simulation, and in the red wing there is an intensity enhancement from Doppler motions that is not obviously visible in the observations.

In Figure 7, we show vertical slices of the corresponding snapshot from the MHD simulation: in the top row, temperature and line-of-sight velocity, in the middle row, density $^{5}$ and magnetic field strength, indicated in black for vertical fields and in red for horizontal fields. For completeness, the velocity field has been over-plotted using arrows in all panels, except in the middle-right panel, where the arrows indicate the direction of the magnetic field in the plane of our slices $(Y-Z)$. For context, we indicate the location of these slices in Figure 6 using a dotted white line.

In the simulation, the emergence of magnetic flux produces a cold up-flowing bubble, where the plasma flow is mostly vertical in the center and turns almost horizontal on the sides. At the boundary with the environment, there seems to be a draining effect, and the plasma flows down. We note that density is higher within the emerging region than in the surroundings because the gas is compressed at the upper boundary of the bubble.

We have computed the contribution function to the intensity and the response function to temperature to assess whether the emission feature observed in the Ca II 8542 intensity profile can be related to temperature enhancements at some height in the atmosphere.

The contribution function $\left(C_{\lambda}\right)$ is computed using the monochromatic source function $\left(S_{\lambda}\right)$, and therefore, it encodes information from many thermodynamical variables that implicitly affect the source function:

$$
C_{\lambda}(z)=S_{\lambda}(z) \cdot e^{-\tau_{\lambda}(z)} \cdot \frac{d \tau_{\lambda}(z)}{d z},
$$

where $\tau_{\lambda}(z)$ is the monochromatic optical depth at height $z$.

The response function $\left(R_{\lambda}\right)$ is computed by directly perturbing the physical quantities in the model, at each layer, and directly quantifying the impact of such a perturbation on the synthetic profile (see, e.g., Landi Degl'Innocenti \& Landi Degl'Innocenti 1977; Ruiz Cobo \& del Toro Iniesta 1992; Socas-Navarro \& Uitenbroek 2004; Orozco Suárez \& Del Toro Iniesta 2007):

$$
R_{\lambda}(z, T)=\frac{\delta I_{\lambda}(z)}{\delta T(z) \delta z},
$$

where $I_{\lambda}$ is the emerging intensity and $T$ is the temperature at height $z$ in the model. Fossum \& Carlsson (2005) proposed an equivalent method to compute the response function, which is numerically more stable, with a slightly different form than Equation (2), that has been used in our study. We applied a perturbation of $\delta T(z)= \pm 0.01 T(z)$.

In the bottom row of Figure 7 we display the $\lambda 8542$ line, computed through the column at the center of the bubble, indicated with a dashed vertical line in the panels above. The blue curve corresponds to the temperature stratification (in the left panel) and to the velocity stratification (in the right panel). The line profile seems to have an emission peak in the red wing; similar to what is observed, but contrary to intuition, the temperature stratification does not exhibit clear enhancements that could be associated with such a peak. Additionally, we

\footnotetext{
Normalized to the average density at each horizontal plane.
} 


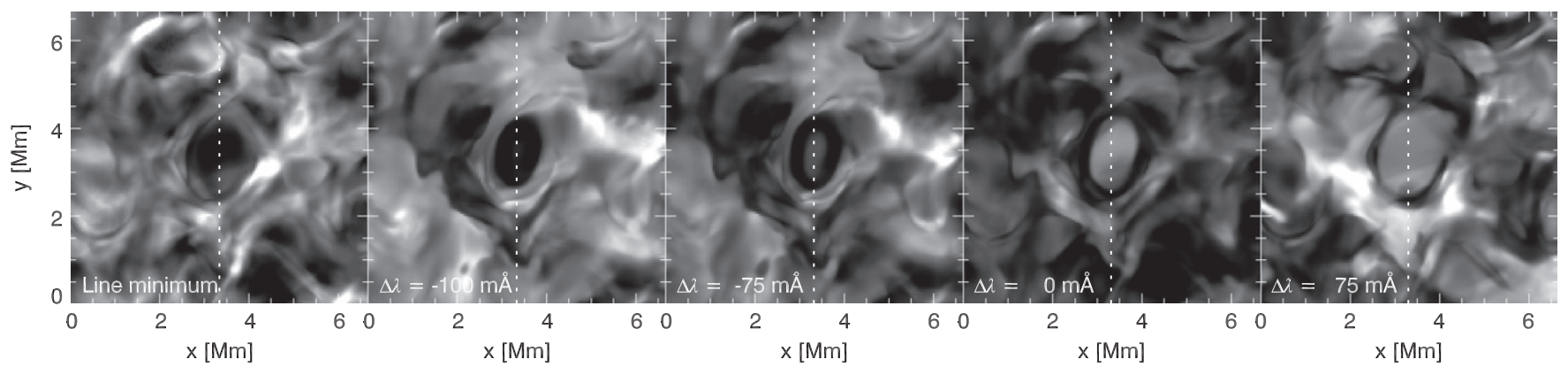

Figure 6. Synthetic intensity images in the $\lambda 8542$ line. The leftmost panel represents the line minimum intensity at each pixel. All the other panels illustrate, from left to right, monochromatic images at $\Delta \lambda=-100,-75,0,75 \mathrm{~m} \AA$, respectively. The vertical dotted line indicates the location of the vertical slices displayed in Figure 7.
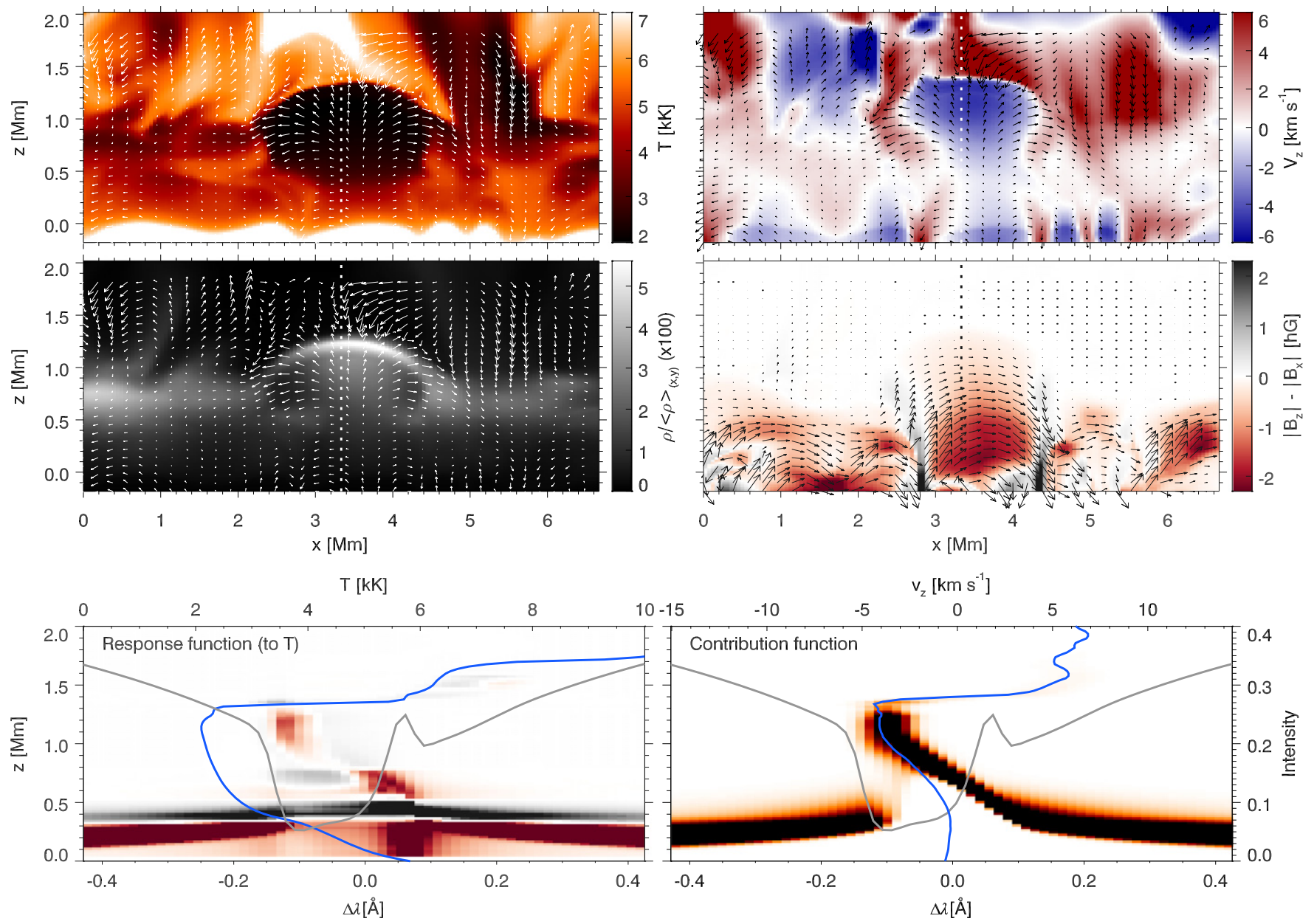

Figure 7. Vertical slice of the temperature (top left), vertical velocity (top right), density (bottom left), and magnetic field topology (bottom right) from a snapshot of the simulation. The magnetic field panel illustrates the vertical component in black and the horizontal component along the $x$-axis in red. The dotted vertical line in the center indicates the location where the response and contribution functions have been computed. The velocity field has been overplotted with arrows in all panels except that showing the magnetic field strength, where the direction of the magnetic field in that plane has been represented instead. Bottom left: response function of the intensity to the temperature. Bottom right: contribution function to the intensity. In both cases, the scale has been clipped to enhance the upper layers. For clarity, the blue curve in the lowermost panels illustrate the corresponding temperature (left panel) and velocity profiles (in the right panel) for that column in the 3D model.

present the response function to the temperature and the contribution function for each wavelength (Uitenbroek 2006a). These two functions quantify how sensitive the spectral line is to different layers of the model; however, the contribution function does not account for radiative coupling of the source function to non-local properties of the atmosphere.

In our calculations, both methods seem to provide similar results: far in the wings, the line is mostly influenced by the photosphere, between 0 and $500 \mathrm{~km}$. Close to the line center, the line is sensitive to higher layers. The blue curve represents the temperature stratification with height (left panel) and the line-of-sight velocity profile (right panel). Their corresponding scales are indicated in the upper axis of each of the panels. Both plots indicate that the core of the line is formed within the rising magnetic region, which is denser than the surroundings in the simulation. 

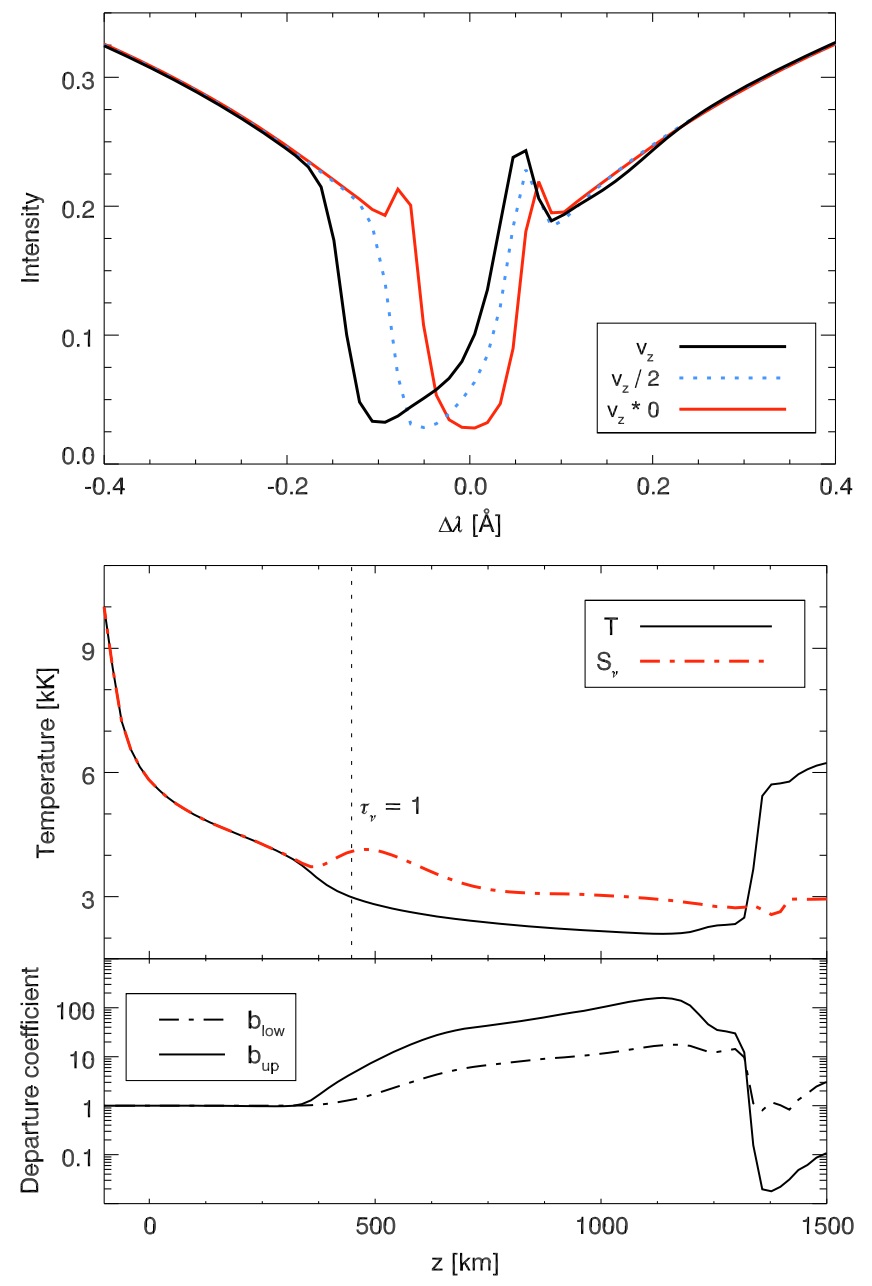

Figure 8. Top: synthetic profiles using the original velocity field (solid black), zero velocities (solid red), and with the velocity field decreased by a factor $\times 2$ (dotted blue). Middle: temperature profile (solid black) inside the magnetic bubble and the corresponding total source function (red dash-dotted) at $\Delta \lambda=+60 \mathrm{m \AA}$. The source function has been converted to radiation temperature for comparison with the temperature profile. Bottom: departure coefficients from LTE populations for the upper level (solid) and the lower level (dash-dotted) of the transition.

The contribution function indicates that the emission peak in the red wing originates at around $450 \mathrm{~km}$ above the surface, coinciding with the base of the bubble. The velocity profile also changes there, with a steeper velocity gradient inside the bubble. The response function shows a complicated sensitivity to changes in temperature within a large range of heights, making it very hard to identify a specific regime responsible for the emission.

Therefore, we attacked the problem with a slightly different approach. To test whether the emission is an effect of Doppler velocities, we ran an experiment where the profile from this column is calculated using the original velocity stratification, with the velocities set to zero, and with the velocities decreased by a factor $\times 2$. The result is illustrated in Figure 8 (top panel). The red curve is computed neglecting the velocity field, and therefore it is symmetric. The emission feature is then visible in both line wings, although there are no temperature enhancements along the line of sight. The plot also demonstrates that the emission continues to be visible when the amplitude of the velocities is half of that in the simulations, but the presence of strong Doppler velocities enhance the emission in the red wing.
The latter is because the gradient of velocities within the bubble (to the blue, as corresponds to up-flowing material) reduces the line opacity in the red wing (see discussion by Scharmer 1984). Therefore, it is the bubble, and not the downflowing material above it, that enhances the emission in the red wing and attenuates it in the blue wing.

To understand the origin of the emission, we inspected the shape of the monochromatic source function and analyzed its dependence on the local temperature profile. In Figure 8 (middle panel) we illustrate the temperature profile of the column and the total source function at $\Delta \lambda=60 \mathrm{m \AA}$, which has been converted to radiation temperature. The source function decouples from the temperature profile at around $350 \mathrm{~km}$ from the surface and peaks at $500 \mathrm{~km}$. Then it decreases again monotonically. At that wavelength, the $\tau_{\nu}=1$ layer coincides with the bump in the source function and therefore the emission feature is formed. However, this behavior is not due to an increase in the temperature (unlike de la Cruz Rodríguez et al. 2013) or in the density profiles, rather the opposite. In the lower panel of Figure 8, we show the departure coefficients for the upper and lower levels of the 8542 line as a function of height. In this case, the departure coefficient for an atomic level is defined using Zwaan's notation (Wijbenga \& Zwaan 1972):

$$
b=\frac{n}{n_{\mathrm{LTE}}},
$$

where $n$ and $n_{\text {LTE }}$ are the actual and LTE populations of that level. At $z=500 \mathrm{~km}$, the upper level departs from LTE more vigorously than the lower level, explaining the peak of the source function at that height.

Uitenbroek (1989) reports that such a behavior can occur because the $\mathrm{Ca}$ II $K$ and the 8542 lines share the upper level, while their lower levels are collisionally coupled. Therefore, the source function of the 8542 line is set by the $K$ line and displays the same temperature sensitivity as $3933 \AA$. We emphasize that the source function in Figure 8 decouples from the temperature profile exactly at the temperature drop produced by the magnetic bubble. Given the behavior observed in the departure coefficients and the shape of the source function, we have no reason to think that this is not the same phenomenon.

We now focus on the polarization signals in our synthetic observations. Figure 9 illustrates some non-consecutive snapshots from the simulation. The left column displays the minimum intensity of the profile at each pixel. In the middle column we made a color composite: in orange we represent $\max \left(\sqrt{Q_{\lambda}^{2}+U_{\lambda}^{2}}\right)$ and in blue $\max \left(\left|V_{\lambda}\right|\right)$. In this way we can visualize where the field is predominantly inclined. The column on the right illustrates Stokes $V$ in the photospheric wing, and provides information about the footpoints of our emerging region. In the beginning of the time series, linear polarization is dominant in the picture, suggesting that the $\lambda 8542$ line is sampling the top of the bubble where the field is mostly inclined. As time goes on and the bubble reaches higher layers in the atmosphere, the imprint of vertical fields becomes stronger on the sides, where the magnetic field connects with the footpoints. This is also consistent with the results from LTE inversions (using the $\mathrm{Fe}_{\mathrm{I}} \lambda 6301 / 6302$ lines) presented in Paper I. Since we are tracking the maximum signal of the Stokes profiles, which can change wavelength due to Doppler motions or a change in the formation regime, the observables 


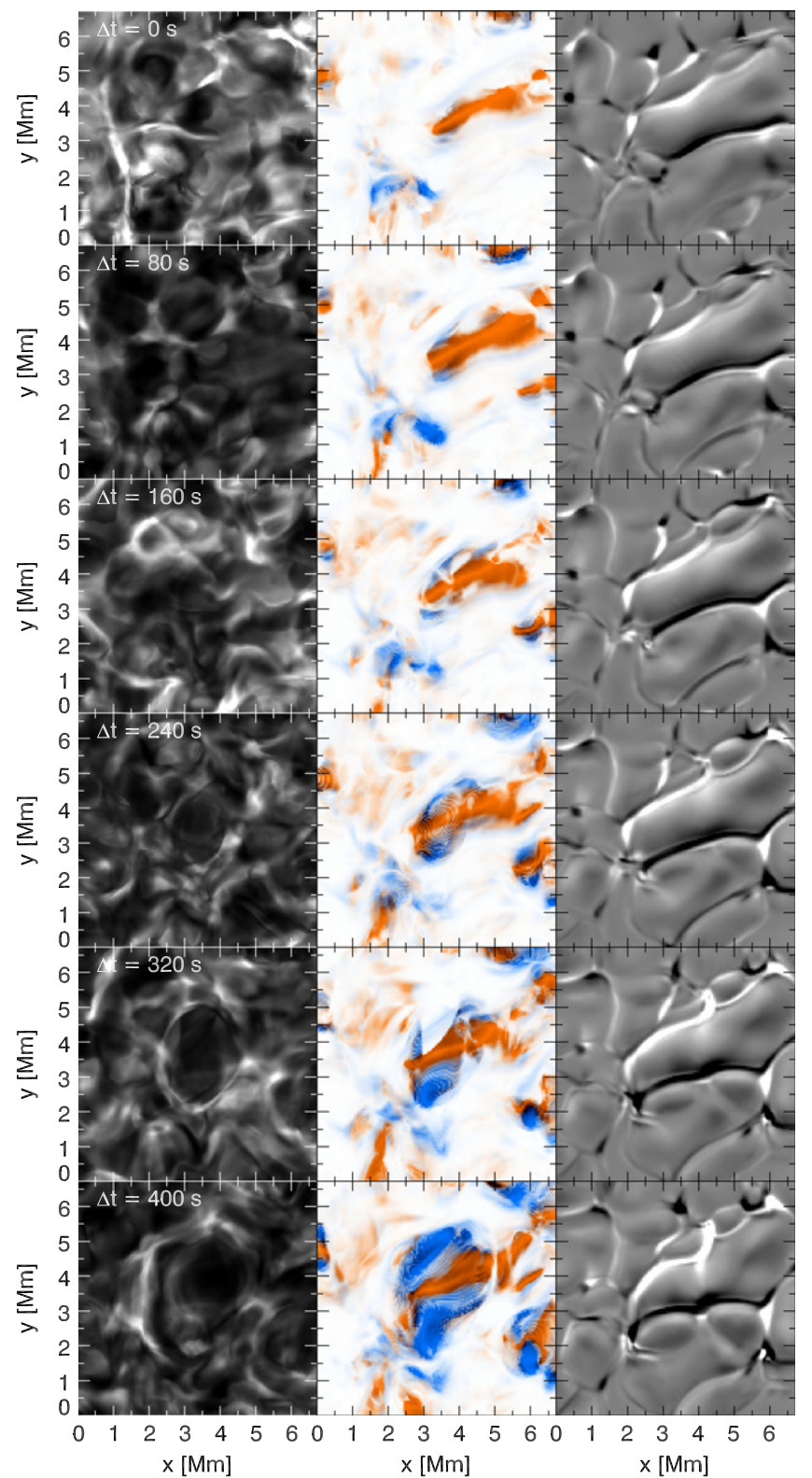

Figure 9. Synthetic observables from our 3D MHD simulation. Left column: intensity image constructed with the minimum intensity of the line at each pixel. Middle column: color composition showing the maximum total linear polarization (orange) and the maximum total circular polatization (blue). To compose the images, we have scaled the total circular and linear polarization to the respective maximum values in the selected subfield. Right column: monochromatic Stokes $V$ at $\Delta \lambda=-1.83 \AA$ from line center. Time increases from top to bottom.

depicted in Figure 9 may be sensitive to slightly different heights in the model.

In our observations we did not detect linear polarization within the magnetic bubble and the circular polarization levels were just above the detection limit, probably because the magnetic field is highly inclined and the sensitivity of Stokes $Q$ and $U$ to the field strength is much lower than for Stokes $V$. Interestingly, we only detected Stokes $V$ signals when the synthetic Stokes I profile showed emission around $\Delta \lambda=60$ $\mathrm{mA}$ (e.g., panel 1 of Figure 10 in Paper I). Therefore, we inspected the polarization levels in the Stokes profiles computed from the MHD simulation. We note that the longitudinal component of the magnetic field inferred from

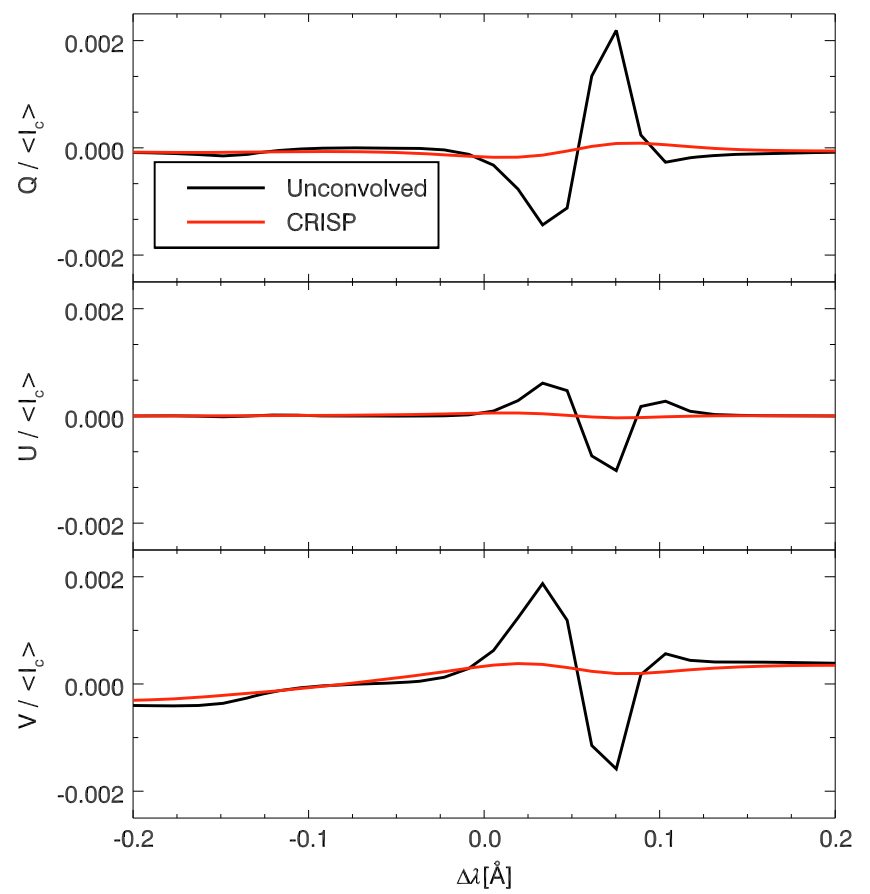

Figure 10. From top to bottom, Stokes $Q, U$, and $V$, convolved with a theoretical CRISP transmission profile (red) and unconvolved (black). The profiles correspond to the same column from the models as it was used in Figure 7. The profiles have been normalized by the continuum intensity of a quiet-Sun average at that wavelength $\left(\left\langle I_{\mathrm{c}}\right\rangle\right)$.

the observations ranges between 100-200 G, and these values are quite similar to those present in the 3D MHD simulation.

The synthetic Stokes $V$ profiles show polarization levels peaking at $0.2 \%$ relative to the continuum intensity for the linear and circular components. We convolved the spectra with a theoretical CRISP transmission profile at $\lambda 8542$ and most of the signal is washed away, showing peak values one order of magnitude lower than the unconvolved ones. However, the profiles computed from our simulation are significantly narrower than the observed profiles (see discussion by Leenaarts et al. 2009; de la Cruz Rodríguez et al. 2012; Rubio da Costa et al. 2015). Therefore, our test with the CRISP transmission profile is likely to be significantly more pessimistic than reality, and it should only be taken as a lower limit. Regions with stronger magnetic fields could produce polarization levels of $\approx 0.1 \%$, which is our detection limit. Figure 10 illustrates this experiment. Incidentally, we note that the model shows Stokes $Q, U$, and $V$ signals only at the position of the emission feature, i.e., the emission brings out polarization signals that would otherwise be hidden. This is also what happens in our observations.

Summarizing, in this section we used a 3D MHD simulation to study the formation of the $\lambda 8542$ within a magnetic bubble emerging into the solar chromosphere. Our analysis reveals that the emission component in the red wing of the line is consistent with an increase of the source function peaking at $z=500 \mathrm{~km}$. The presence of a strong upflow in the bubble makes the profile highly asymmetric, hides the emission feature in the blue wing, and makes the emission in the red wing stronger.

\section{CONCLUSIONS AND DISCUSSION}

In this paper we studied granular-sized flux emergence and the formation of the $\lambda 8542$ line within an emerging bubble. To 
this end, we performed a multi-pronged analysis using a 3D MHD simulation and non-LTE inversions of real observations. This study has been driven by two main goals: to explain the observed $\lambda 8542$ profiles and to understand the structuring of atmospheric parameters within flux emerging regions.

We performed non-LTE inversions of our observational data, obtaining 3D estimates of the temperature and the line-ofsight velocity. Our inferred maps show the temporal evolution of the temperature stratification as the bubble emerges from the photosphere and protrudes into the lower chromosphere. The magnetic bubble leaves a cold imprint in the temperature maps, which is first visible in the middle/upper photosphere and, with a temporal delay, in the lower chromosphere. This result is consistent with the analysis performed in Paper I, using the wings of the 8542 line and LTE inversions of the observed photospheric lines. Our inversions also reveal two opposite polarity patches that separate during the ascent of the bubble. They seem to trace the more vertical field of the footpoints in the upper photosphere, where the bubble is visible. The inferred longitudinal field peaks at $\pm 140 \mathrm{G}$.

Our analysis of the 3D MHD simulation has provided valuable information in understanding the peculiarities of the spectral profiles that are observed within the emerging region. The emission feature that is present in the red wing of the $\lambda 8542$ line can be explained by the coupling of the $\lambda 8542$ source function with the Ca II $K$ line, which can pump electrons to the upper level of the transition, and by the velocity gradient within the bubble, which enhances the emission peak in the red wing and attenuates the emission in the blue wing. These mechanisms naturally explain the emission feature in the red wing of the line, given that the bubble is colder than its surroundings, but we have not been able to assess whether the interactions between the emerging flux and the canopy of fibrils also has a contribution to that emission.

In the 3D MHD simulation, the magnetic region emerged without the presence of an organized large-scale magnetic field in the chromosphere and could therefore reach higher layers. In the observations, the bubble seems to get stuck once it reaches the canopy of fibrils. We believe this is the reason why the bubble is visible at the line center in our synthetic Ca II 8542 observations, but not in the observations (except perhaps for weak hints of its presence).

We identify signatures of chromospheric heating in the lower chromosphere, although we have not been able to identify the heating mechanism that produces these signatures. Also, we have not been able to assess whether the magnetic region continues rising into the upper chromosphere or if it is destroyed by interaction with the existing chromospheric magnetic field. These aspects must be addressed in future studies, perhaps including lines that are sensitive to the upper chromosphere and transition region (like the $\mathrm{Mg}$ II $h$ and $k$ lines, see, e.g., Leenaarts et al. 2013 and the Si Iv lines) and to the corona. This will be the subject of Paper III of the present series (A. Ortiz et al. 2015, in preparation), where we will analyze simultaneous observations of flux emergence events with the SST and the IRIS space mission (De Pontieu et al. 2014b).

The authors thank J. Leenaarts and L. Rouppe van der Voort for illuminating discussions. J. de la Cruz Rodríguez acknowledges financial support from the CнRомовs project funded by the Knut and Alice Wallenberg Foundation. L. Bellot Rubio is funded by grants AYA2012-39636-C06-05 and ESP2013-
47349-C6-1-R of the Spanish Ministerio de Economía y Competitividad, including a percentage from European FEDER funds. The Swedish $1 \mathrm{~m}$ Solar Telescope is operated on the island of La Palma by the Institute for Solar Physics of Stockholm University in the Spanish Observatorio del Roque de los Muchachos of the Instituto de Astrofísica de Canarias. The radiative transfer computations and inversions were performed on resources provided by the Swedish National Infrastructure for Computing (SNIC) at the National Supercomputer Centre (Linköping University) and at the High Performance Computing Center North (Umeå University) with project id snic2014-1-273. Part of this study was discussed in meetings at the International Space Science Institute (ISSI) in Switzerland. This research has made use of NASA's Astrophysics Data System Bibliographic Services.

\section{REFERENCES}

Archontis, V., \& Hansteen, V. 2014, ApJL, 788, L2

Auer, L. 2003, in ASP Conf. Ser. 288, Stellar Atmosphere Modeling, ed. I. Hubeny, D. Mihalas \& K. Werner (San Francisco, CA: ASP), 3

Beck, C., \& Rezaei, R. 2009, A\&A, 502, 969

Carlin, E. S., \& Asensio Ramos, A. 2015, ApJ, 801, 16

Carlin, E. S., Manso Sainz, R., Asensio Ramos, A., \& Trujillo Bueno, J. 2012, ApJ, 751, 5

Carlsson, M. 1986, UppOR, 33

Castelli, F., \& Hubrig, S. 2004, A\&A, 421, L1

Chae, J., Park, H.-M., Ahn, K., et al. 2013, SoPh, 288, 89

Cheung, M. C. M., Schüssler, M., \& Moreno-Insertis, F. 2007, A\&A, 461, 1163

Cheung, M. C. M., Schüssler, M., Tarbell, T. D., \& Title, A. M. 2008, ApJ, 687,1373

Cowley, C. R., \& Hubrig, S. 2005, A\&A, 432, L21

Cowley, C. R., Hubrig, S., Castelli, F., González, J. F., \& Wolff, B. 2007, MNRAS, 377, 1579

Danilovic, S., Beeck, B., Pietarila, A., et al. 2010, ApJL, 723, L149

de la Cruz Rodríguez, J., De Pontieu, B., Carlsson, M., \& Rouppe van der Voort, L. H. M. 2013, ApJL, 764, L11

de la Cruz Rodríguez, J., Kiselman, D., \& Carlsson, M. 2011, A\&A, 528, A113 de la Cruz Rodríguez, J., Löfdahl, M. G., Sütterlin, P., Hillberg, T., \& Rouppe van der Voort, L. 2015, A\&A, 573, A40

de la Cruz Rodríguez, J., \& Piskunov, N. 2013, ApJ, 764, 33

de la Cruz Rodríguez, J., Socas-Navarro, H., Carlsson, M., \& Leenaarts, J. 2012, A\&A, 543, A34

De Pontieu, B., Rouppe van der Voort, L., McIntosh, S. W., et al. 2014a, Sci, 346, 1255732

De Pontieu, B., Title, A. M., Lemen, J. R., et al. 2014b, SoPh, 289, 2733

Fossum, A., \& Carlsson, M. 2005, ApJ, 625, 556

Galsgaard, K., Archontis, V., Moreno-Insertis, F., \& Hood, A. W. 2007, ApJ, 666,516

Galsgaard, K., \& Nordlund, Å. 1996, JGR, 101, 13445

Gošić, M., Bellot Rubio, L. R., Orozco Suárez, D., Katsukawa, Y., \& del Toro Iniesta, J. C. 2014, ApJ, 797, 49

Gudiksen, B. V., Carlsson, M., Hansteen, V. H., et al. 2011, A\&A, 531, A154 Gudiksen, B. V., \& Nordlund, Å. 2005, ApJ, 618, 1020

Guglielmino, S. L., Bellot Rubio, L. R., Zuccarello, F., et al. 2010, ApJ, 724,1083

Hansteen, V., De Pontieu, B., Carlsson, M., et al. 2014, Sci, 346, 1255757

Henriques, V. M. J. 2012, A\&A, 548, A114

Khomenko, E., \& Collados, M. 2012, ApJ, 747, 87

Klimchuk, J. A. 2006, SoPh, 234, 41

Landi Degl'Innocenti, E., \& Landi Degl'Innocenti, M. 1977, A\&A, 56, 111

Leenaarts, J., Carlsson, M., Hansteen, V., \& Rouppe van der Voort, L. 2009, ApJL, 694, L128

Leenaarts, J., Carlsson, M., Hansteen, V., \& Rutten, R. J. 2007, A\&A, 473, 625

Leenaarts, J., Carlsson, M., \& Rouppe van der Voort, L. 2012, ApJ, 749, 136

Leenaarts, J., de la Cruz Rodríguez, J., Kochukhov, O., \& Carlsson, M. 2014, ApJL, 784, L17

Leenaarts, J., Pereira, T. M. D., Carlsson, M., Uitenbroek, H., \& De Pontieu, B. 2013, ApJ, 772, 90

Lites, B. W., Kubo, M., Socas-Navarro, H., et al. 2008, ApJ, 672, 1237

Manso Sainz, R., \& Trujillo Bueno, J. 2010, ApJ, 722, 1416 
Mariska, J. T. (ed.) 1992, The Solar Transition Region (Vol. 23; Cambridge: Cambridge Univ. Press)

Martínez González, M. J., \& Bellot Rubio, L. R. 2009, ApJ, 700, 1391

Martínez-Sykora, J., De Pontieu, B., \& Hansteen, V. 2012, ApJ, 753, 161

Martinez-Sykora, J., De Pontieu, B., Hansteen, V. H., \& Carlsson, M. 2015, RSPTA, 373, 40268

Martínez-Sykora, J., Hansteen, V., \& Carlsson, M. 2008, ApJ, 679, 871

Martínez-Sykora, J., Hansteen, V., \& Carlsson, M. 2009, ApJ, 702, 129

Orozco Suárez, D., \& Del Toro Iniesta, J. C. 2007, A\&A, 462, 1137

Ortiz, A., Bellot Rubio, L. R., Hansteen, V. H., de la Cruz Rodríguez, J., \& Rouppe van der Voort, L. 2014, ApJ, 781, 126

Pietarila, A., \& Harvey, J. W. 2013, ApJ, 764, 153

Piskunov, N. E., Kupka, F., Ryabchikova, T. A., Weiss, W. W., \& Jeffery, C. S. 1995, A\&AS, 112, 525

Rubio da Costa, F., Kleint, L., Petrosian, V., Sainz Dalda, A., \& Liu, W. 2015, ApJ, 804, 56

Ruiz Cobo, B., \& del Toro Iniesta, J. C. 1992, ApJ, 398, 375

Ryabchikova, T., Kochukhov, O., \& Bagnulo, S. 2008, A\&A, 480, 811

Scharmer, G. B. 1984, in Accurate Solutions to Non-LTE Problems using Approximate Lambda Operators (Cambridge and New York: Cambridge Univ. Press), 173

Scharmer, G. B., de la Cruz Rodriguez, J., Sütterlin, P., \& Henriques, V. M. J. 2013, A\&A, 553, A63

Scharmer, G. B., Narayan, G., Hillberg, T., et al. 2008, ApJL, 689, L69
Schmieder, B., Archontis, V., \& Pariat, E. 2014, SSRv, 186, 227

Skartlien, R. 2000, ApJ, 536, 465

Socas-Navarro, H., de la Cruz Rodriguez, J., Asensio Ramos, A., Trujillo Bueno, J., \& Ruiz Cobo, B. 2015, A\&A, 577, A7

Socas-Navarro, H., \& Trujillo Bueno, J. 1997, ApJ, 490, 383

Socas-Navarro, H., Trujillo Bueno, J., \& Ruiz Cobo, B. 2000, ApJ, 530, 977

Socas-Navarro, H., \& Uitenbroek, H. 2004, ApJL, 603, L129

Solanki, S. K., Lagg, A., Woch, J., Krupp, N., \& Collados, M. 2003, Natur, 425,692

Steiner, O., Hauschildt, P. H., \& Bruls, J. 2001, A\&A, 372, L13

Tian, H., DeLuca, E. E., Cranmer, S. R., et al. 2014, Sci, 346, 1255711

Tortosa-Andreu, A., \& Moreno-Insertis, F. 2009, A\&A, 507, 949

Uitenbroek, H. 1989, A\&A, 213, 360

Uitenbroek, H. 2006a, in ASP Conf. Ser. 354, Solar MHD Theory and Observations: A High Spatial Resolution Perspective, ed. J. Leibacher, R. F. Stein \& H. Uitenbroek (San Francisco, CA: ASP), 313

Uitenbroek, H. 2006b, ApJ, 639, 516

van Noort, M. 2012, A\&A, 548, A5

van Noort, M., Rouppe van der Voort, L., \& Löfdahl, M. G. 2005, SoPh, 228,191

Watanabe, H., Bellot Rubio, L. R., de la Cruz Rodríguez, J., \& Rouppe van der Voort, L. 2012, ApJ, 757, 49

Wedemeyer-Böhm, S., \& Carlsson, M. 2011, A\&A, 528, A1

Wijbenga, J. W., \& Zwaan, C. 1972, SoPh, 23, 265 